\title{
Correspondence between Surface Tension Estimated by a Surface Thermodynamic Model and Number of Bubbles in the Vicinity of the Surface of Steel Products in Continuous Casting Process
}

\author{
Taishi MATSUSHITA, ${ }^{11 *}$ Kusuhiro MUKAI') and Masafumi ZEZE ${ }^{3)}$ \\ 1) Department of Materials and Manufacturing-Casting, School of Engineering, Jönköping University, P.O. Box 1026, SE-551 11 \\ Jönköping, Sweden. $\quad$ 2) Professor emeritus, Department of Materials Science and Engineering, Kyushu Institute of \\ Technology, 1-1, Sensui-cho, Tobata, Kitakyushu, 804-8550 Japan. $\quad 3$ 3) Yawata R\&D Laboratory, Nippon Steel \\ Corporation, 1-1, Tobihata-cho, Tobata, Kitakyushu, 804-8501 Japan. Now at Nippon Steel \& Sumitomo Metal Corporation.
}

(Received on July 12, 2012; accepted on September 20, 2012)

\begin{abstract}
Surface tensions of low carbon slabs and 16 mass\%Cr stainless steel were estimated using a surface thermodynamic model proposed by Mukai et al. As an application of the model, an index to evaluate the driving force for the fine bubble entrapment by the solidifying shell, the Mukai-value, $M$, was calculated from the surface tension values. The relationship between Mukai-value and number of entrapped bubbles was discussed. A linear relationship was found between the number of captured bubbles and Mukaivalue. In the previous work, the Mukai-value was used as a relative scale to evaluate the driving force for the movement of bubbles. However, by calculating the $M$ from the surface tension values by the surface thermodynamic model, physically reasonable Mukai-values could be obtained.
\end{abstract}

KEY WORDS: surface tension; prediction; surface thermodynamic model; Mukai-value; bubble entrapment.

\section{Introduction}

In the steelmaking process, surface tension of multicomponent dilute solution system is regarded as significant. However, to the knowledge of the present authors, efficient and reasonable methods to estimate or predict the surface tension have not been established so far. Hence, the measurements have to be done in each case.

A goal of the present paper is to predict the surface tension of multicomponent dilute metallic solutions. In this paper, an attempt was made to predict the surface tension of low carbon steel and 16 mass $\% \mathrm{Cr}$ stainless steel $(16 \mathrm{Cr}$ SUS), by a recently developed surface tension model by some of the present authors. ${ }^{1)}$ The surface tension of multicomponent system has been predicted using parameters derived from surface tension values in literature.

Another goal of the present paper is to suggest a guideline to suppress the entrapment of the fine gas bubbles and/or nonmetallic inclusions by the solidifying shell during continuous casting. The entrapment of the fine particles at solidifying interface is a serious problem since it becomes a cause of defects. Such defects will lower the mechanical properties and it will become surface defects after the machining. Hence, it is desired to suppress the entrapment of such fine particles to improve the quality of steel product.

A mechanism of the fine particle entrapment was proposed by some of the present authors. ${ }^{2,3)}$ In the previous works, it has become apparent that fine particles in the mol-

* Corresponding author: E-mail: taishi.matsushita@jth.hj.se DOI: http://dx.doi.org/10.2355/isijinternational.53.18 ten steel such as bubble are entrapped by solidifying interface during the solidification and remained in the final products. It is concluded that the surface tension gradient in the vicinity of solidification interface is a controlling factor of the bubble entrapment.

In the present study, the relationship between the number of entrapped bubbles during the casting process and an index to evaluate the driving force of the entrapment, Mukai-value, $M$, was investigated. Feasibility of the suppression of the fine particle entrapment at solidifying interface was discussed based on the above-mentioned mechanism.

\subsection{Prediction of Surface Tension of Multicomponent Dilute Solution (Surface Thermodynamic Model) ${ }^{1)}$}

In the present work, attempt was made to estimate the surface tension of commercial steels based on a surface thermodynamic model proposed by Mukai et al. ${ }^{1)}$ The calculated surface tension values are used to calculate the Mukai-value which is mentioned in the next section. An outline of the surface thermodynamic model for the estimation of surface tension is briefly summarized below:

A thermodynamic formula has been derived to describe (or predict) the surface tension of binary and multi-component systems by some of the present authors. ${ }^{1)}$ The starting point of the present model for the estimation of surface tension is the total Helmholtz energy of the system, $F$

$$
F\left(T, V, A, n_{\mathrm{i}}\right)=-P^{l} V^{l}+\sigma A+\sum_{\mathrm{i}=1}^{r} \mu_{\mathrm{i}} n_{\mathrm{i}}
$$

where $P^{l}$ is the pressure of liquid phase, $V^{l}$ is the volume of 
liquid phase, $\sigma$ is the surface tension, $\mu_{\mathrm{i}}$ is the chemical potential of component i, $n_{\mathrm{i}}$ is the number of moles of component $\mathrm{i}$ in the system, viz., $n_{\mathrm{i}}=n_{\mathrm{i}}^{\mathrm{b}}+n_{\mathrm{i}}^{\mathrm{s}}\left(n_{\mathrm{i}}^{\mathrm{b}}\right.$ : the number of moles of component $\mathrm{i}$ in the bulk phase, $n_{\mathrm{i}}^{\mathrm{s}}$ the number of moles of component $i$ in (or at) the surface (area)).

It was found that the surface tension could be described by the difference of "logarithm of the activity coefficient of component $i$ of the liquid phase including surface" and "logarithm of the activity coefficient of component $i$ of the liquid bulk phase". It was attempted to describe the surface tension for binary and ternary dilute iron alloys as a function of concentration at a certain temperature. In the case of ternary dilute iron alloy, the activity coefficient of solute could be estimated by the polynomial equation as a function of concentration with interaction parameters. In the same way, the surface tension of ternary dilute iron alloys was described by the polynomial equation as a function of concentration. For example, the surface tension of Fe-i-j system could be described as follows: ${ }^{1)}$

$$
\begin{aligned}
\sigma=\sigma_{\mathrm{Fe}}+k & \frac{M_{\mathrm{Fe}}\left(\mu_{\underline{\mathrm{i}}}^{\ominus^{\prime}}-\mu_{\underline{\mathrm{i}}}^{\ominus}\right)}{100 M_{\mathrm{i}} R T} w_{\mathrm{i}}+\frac{2.303 M_{\mathrm{Fe}}}{100 M_{\mathrm{i}}}\left(e_{\mathrm{i}}^{\mathrm{i}^{\prime}}-e_{\mathrm{i}}^{\mathrm{i}}\right) w_{\mathrm{i}}^{2} \\
& +\frac{2.303 M_{\mathrm{Fe}}}{100}\left\{\frac{\left(e_{\mathrm{i}}^{\mathrm{j}^{\prime}}-e_{\mathrm{i}}^{\mathrm{j}}\right)}{M_{\mathrm{i}}}+\frac{\left(e_{\mathrm{j}}^{\mathrm{i}^{\prime}}-e_{\mathrm{j}}^{\mathrm{i}}\right)}{M_{\mathrm{j}}}\right\} w_{\mathrm{i}} w_{\mathrm{j}} \\
& \left.+\frac{M_{\mathrm{Fe}}\left(\mu_{\underline{\mathrm{j}}}^{\ominus^{\prime}}-\mu_{\underline{\mathrm{j}}}^{\ominus}\right)}{100 M_{\mathrm{j}} R T} w_{\mathrm{j}}+\frac{2.303 M_{\mathrm{Fe}}}{100 M_{\mathrm{j}}}\left(e_{\mathrm{j}}^{\mathrm{j}^{\prime}}-e_{\mathrm{j}}^{\mathrm{j}}\right) w_{\mathrm{j}}^{2}\right]
\end{aligned}
$$

where $k=\left(R T / A_{0}\right) \cdot 10^{7}, A_{0}$ is the surface area of the system with one mole solution, $\sigma_{\mathrm{Fe}}$ is the surface tension of pure iron at temperature $T, w_{\mathrm{i}}$ is the mass percent of the component i, $M_{\mathrm{i}}$ is the atomic weight of component $\mathrm{i}, \mu_{\mathrm{i}}^{\ominus}$ is the chemical potential of $i$ in the bulk for the standard state at $1 \mathrm{mass} \% w_{\mathrm{i}}$ for a dilute, ideal solution, $R$ is the gas constant, $e_{\mathrm{i}}^{\mathrm{j}}$ is the interaction parameter and prime denotes the property of the phase including surface phase. For the simplicity, all coefficients may be replaced by a parameter $P$ as follows:

$$
\sigma=\sigma_{\mathrm{Fe}}+P_{\mathrm{i}} w_{\mathrm{i}}+P_{\mathrm{ii}} w_{\mathrm{i}}^{2}+P_{\mathrm{j}} w_{\mathrm{j}}+P_{\mathrm{ij}} w_{\mathrm{i}} w_{\mathrm{j}}+P_{\mathrm{jj}} w_{\mathrm{j}}^{2}
$$

As shown in the equation, the surface tension can be described by surface tension of pure element $\left(\sigma_{\mathrm{Fe}}\right)$, firstorder terms, second-order terms and cross terms of mass percent of each element. By a critical survey of the data on the parameters, $P_{\mathrm{i}}, P_{\mathrm{j}}$ and $P_{\mathrm{ij}}$, for ternary system and by extending Eq. (2), it is also possible to predict the surface tension of higher order multicomponent dilute iron alloys.

In this paper, an attempt was made to predict the surface tension of low carbon steel and 16 mass $\% \mathrm{Cr}$ stainless steel (16Cr SUS) by using this model. The parameters, $P$, can be calculated using thermodynamic parameters as shown in Eq. (2). However it is practically more difficult to obtain such thermodynamic parameters by experiments or calculation than measure the surface tension. Hence these parameters were obtained by fitting the experimental surface tension values against concentration in the present paper.

\subsection{Entrapment Behavior of Fine Bubbles in Continu- ous Casting Process}

As an application of above mentioned surface thermodynamic model $^{1)}$ for surface tension, the entrapment behavior of the fine bubbles by the solidifying shell in the continuous casting slabs could be discussed since the Mukai-value, $M$, could be calculated using the surface tension values. The mechanism of the fine particles entrapment by solidifying interface has been elucidated by Mukai and his group. They have concluded that a mechanism of the entrapment of fine particles by solidifying interface is due to the surface (interfacial) tension gradient which is generated around the surface of the bubble (or around the interface of the particle) caused by a solute concentration gradient., ${ }^{2,3)}$ The particle should be moved toward solidifying interface when the surface (interfacial) tension of bulk side of the particle is higher than that of solidifying interface side (Fig. 1).

In the continuous casting, the bubbles in the melts will be entrapped at solidifying interface during the casting. Therefore it might be possible to estimate when and where the bubbles are captured by investigating the location of the pores after the casting. The bubbles which entrapped at an earlier stage of the solidification will be remained near the surface and it might become a cause of surface defects. On the other hand, the bubbles which captured at a later stage will be remained far from the surface. It might lead the lower mechanical properties and surface defects after the machining, which are not desired. According to the mechanism, the entrapment of the bubbles can be suppressed by introducing positive surface tension gradient toward the solidification interface. In the present work, an attempt was made to find out the feasibility of the suppression of bubble capturing based on the mechanism.

The index to estimate the driving force for the fine bubble entrapment, Mukai-value, can be described as follows:. ${ }^{2,3)}$

$$
M=\Sigma \mathrm{d} \sigma / \mathrm{d} w_{\mathrm{i}}\left(1-1 / k_{0}{ }^{\mathrm{i}}\right) w_{\mathrm{i}}
$$

or

$$
M=\sigma\left(w_{\mathrm{i}}\right)-\sigma\left(w_{\mathrm{i}} / k_{\mathrm{o}}{ }^{\mathrm{i}}\right)
$$

where

$\sigma$ is the surface tension.

$w_{\mathrm{i}}$ is the concentration of component $\mathrm{i}$ in the bulk.

$k_{0}{ }^{i}$ is the equilibrium partition coefficient of component $\mathrm{i}$. $w_{\mathrm{i}} / \mathrm{k}_{0}{ }^{\mathrm{i}}$ is the solute concentration at the solid-liquid interface.

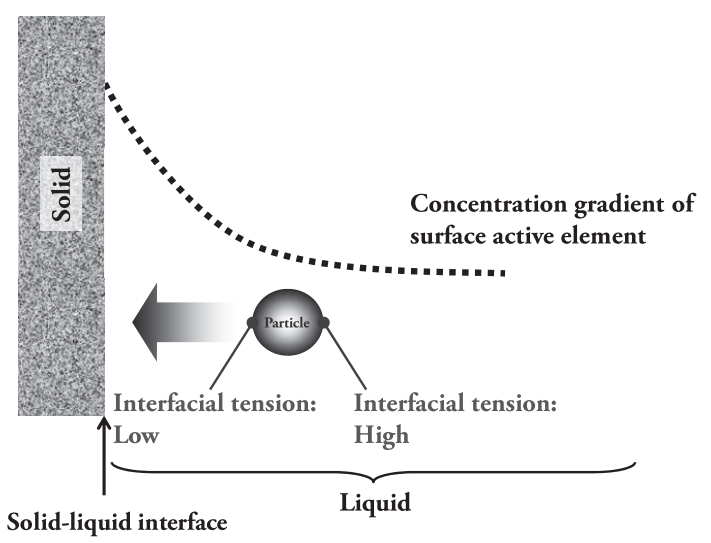

Fig. 1. Motion of a fine particle at solid-liquid interface. 
$\sigma\left(w_{\mathrm{i}}\right)$ is the surface (interfacial) tension with the steel composition at the bulk phase.

$\sigma\left(w_{\mathrm{i}} / k_{\mathrm{o}}{ }^{\mathrm{i}}\right)$ is the surface (interfacial) tension with the steel composition at the solidifying interface.

As can be seen from the Eq. (5), Mukai-value corresponds to the surface tension difference due to the composition difference between bulk and solid-liquid interface. The surface tension gradient in the boundary layer could be obtained by dividing the Mukai-value by the concentration boundary layer thickness. Even if the boundary layer thickness is unknown, it is possible to compare the driving forces for the fine bubble entrapment by composition difference with Mukai-value if it is assumed that the concentration boundary layer thickness is same. The boundary layer thickness can be regarded as same under the same casting conditions.

In the present study, the surface thermodynamic model for surface tension which is described in the previous section is used to estimate the $\sigma\left(w_{\mathrm{i}}\right)$ and $\sigma\left(w_{\mathrm{i}} / \mathrm{k}_{\mathrm{o}}{ }^{\mathrm{i}}\right)$ in the Eq. (5) for the calculation of Mukai-value, $M$.

\subsection{The Number of the Captured Bubbles in Continu- ous Casting Strand}

To study the relationship between Mukai-value and the number of captured bubbles, the number of captured bubbles were counted for six different stainless steels $(16 \mathrm{Cr}$ SUS) and three different low carbon steels which cast in continuous casting by Mukai et al. ${ }^{2-4)}$ The composition of each steel grade are shown in Tables $\mathbf{1}$ and $\mathbf{2}$.

The $16 \mathrm{Cr}$ SUS shown in Table 1 were cast using the No. 1 vertical-bending type continuous caster of Yawata works, Nippon Steel Corporation under about the same casting condition. ${ }^{4)}$ Then, the number of bubbles with radius larger than $0.25 \mathrm{~mm}$ in the stainless steel slab $(0-125 \mathrm{~mm}$ from the surface) was counted from the X-ray images ${ }^{2)}$ since such bubbles have the potential to become harmful defects.

All low carbon steels shown in Table 2 were cast using the No. 2 vertical-bending type continuous caster of Yawata works, Nippon Steel Corporation under about the same casting condition including flow rate of argon, casting speed and

Table 1. Composition of each stainless steel grades (mass\%).

\begin{tabular}{lcccccc}
\hline \multirow{2}{*}{ Element } & $\begin{array}{c}\text { Low } \\
\text { Al (1) }\end{array}$ & $\begin{array}{c}\text { Low } \\
\text { Al }(2)\end{array}$ & $\begin{array}{c}\text { Low } \\
\text { Al (3) }\end{array}$ & $\begin{array}{c}\text { Medium } \\
\text { Al (1) }\end{array}$ & $\begin{array}{c}\text { Medium } \\
\text { Al (2) }\end{array}$ & High Al \\
\hline $\mathrm{Fe}$ & Balance & Balance & Balance & Balance & Balance & Balance \\
$\mathrm{C}$ & 0.065 & 0.065 & 0.064 & 0.075 & 0.049 & 0.047 \\
$\mathrm{Si}$ & 0.283 & 0.285 & 0.283 & 0.289 & 0.559 & 0.300 \\
$\mathrm{Mn}$ & 0.580 & 0.596 & 0.580 & 0.606 & 0.490 & 0.187 \\
$\mathrm{P}$ & 0.025 & 0.025 & 0.025 & 0.026 & 0.031 & 0.025 \\
$\mathrm{~S}$ & 0.0085 & 0.0087 & 0.0071 & 0.0014 & 0.0021 & 0.0013 \\
$\mathrm{Soluble} \mathrm{Al} *$ & 0.0060 & 0.0019 & 0.0023 & 0.0890 & 0.0512 & 0.1290 \\
$\mathrm{Cr}$ & 15.91 & 15.90 & 15.90 & 16.22 & 16.24 & 15.99 \\
$\mathrm{Mo}$ & 0.000 & 0.000 & 0.000 & 0.000 & 0.940 & 0.000 \\
$\mathrm{~N}$ & 0.0363 & 0.0331 & 0.0324 & 0.0284 & 0.0030 & 0.0111 \\
Free O * & 0.0020 & 0.0045 & 0.0035 & 0.0005 & 0.0004 & 0.0003 \\
\hline
\end{tabular}

* The concentration of free oxygen and soluble aluminium was calculated from the aluminium deoxidation equilibrium in the steel. Interaction of the other elements was also considered. width of the slab. Then, the number of bubbles, which radius is larger than $0.1 \mathrm{~mm}$, in the sub-surface layer of low carbon steel slab $(0-15 \mathrm{~mm})$ was counted. ${ }^{3)}$

The number of the captured bubbles for low carbon steel and stainless steel are summarized in Tables 3 and 4 . The number of bubbles per $\mathrm{m}^{2}$ was counted for the low carbon steel and the number of bubble per $\mathrm{cm}^{3}$ was counted from the X-ray images for the $16 \mathrm{Cr}$ SUS. The number of bubbles were counted in a different way. However the Mukai-value is a parameter to evaluate the driving force of the movement of fine particle toward solid liquid interface due to the surface tension difference. Therefore the difference of the measurement method is out of question so far as the comparison is made with the number of bubbles which is counted by the same method.

\subsection{Parameters for the Surface Tension of Multicom- ponent Dilute Solution (Low Carbon Steel)}

An equation to describe the surface tension was derived using the surface thermodynamic model $^{1)}$ to calculate surface tension and Mukai-value of present steel grades. For the prediction of surface tension, the influence of strong sur-

Table 2. Composition of each low carbon steel grades (mass\%).

\begin{tabular}{lccc}
\hline \multicolumn{1}{c}{ Element } & Low carbon (1) & Low carbon (2) & Low carbon (3) \\
\hline $\mathrm{Fe}$ & Balance & Balance & Balance \\
$\mathrm{C}$ & 0.037 & 0.013 & 0.002 \\
$\mathrm{Si}$ & 0.009 & 0.009 & 0.010 \\
$\mathrm{Mn}$ & 0.141 & 0.182 & 0.133 \\
$\mathrm{P}$ & 0.015 & 0.008 & 0.008 \\
$\mathrm{~S}$ & 0.0088 & 0.0138 & 0.0142 \\
$\mathrm{Soluble} \mathrm{Al} \mathrm{*}$ & 0.0288 & 0.0533 & 0.0507 \\
$\mathrm{Ti}$ & 0.0000 & 0.0009 & 0.0586 \\
$\mathrm{~N}$ & 0.0023 & 0.0035 & 0.0029 \\
Free O & 0.0001 & 0.0001 & 0.0001 \\
\hline
\end{tabular}

* The concentration of free oxygen and soluble aluminium was calculated from the aluminium deoxidation equilibrium in the steel. Interaction of the other elements was also considered.

Table 3. Number of captured bubbles per $\mathrm{m}^{2}$ (Low carbon steel).

\begin{tabular}{cc}
\hline & Number of bubbles \\
\hline LC(1) & 42 \\
LC(2) & 100 \\
LC(3) & 108 \\
\hline
\end{tabular}

Table 4. Number of captured bubbles per $\mathrm{cm}^{3}$ (Stainless steel).

\begin{tabular}{lc}
\hline & Number of bubbles \\
\hline Low Al (1) & 0.314 \\
Low Al (2) & 0.302 \\
Low Al (3) & 0.300 \\
Medium Al (1) & 0.119 \\
Medium Al (2) & 0.042 \\
High Al & 0.025
\end{tabular}


face active elements, sulfur, oxygen and nitrogen, were considered. In addition, influence of titanium, niobium and boron were taken into account in the present paper. According to the model for surface tension, the surface tension of $\mathrm{Fe}-\mathrm{N}-\mathrm{O}-\mathrm{S}-\mathrm{Ti}-\mathrm{Nb}-\mathrm{B}$ system at a certain temperature, $\sigma$, could be expressed as follows by extending Eq. (3):

$$
\begin{aligned}
\sigma= & \sigma_{\mathrm{Fe}}+P_{\mathrm{N}} w_{\mathrm{N}}+P_{\mathrm{O}} w_{\mathrm{O}}+P_{\mathrm{S}} w_{\mathrm{S}}+P_{\mathrm{NN}} w_{\mathrm{N}}^{2}+P_{\mathrm{OO}} w_{\mathrm{O}}^{2}+ \\
& P_{\mathrm{SS}} w_{\mathrm{S}}^{2}+P_{\mathrm{NO}} w_{\mathrm{N}} w_{\mathrm{O}}+P_{\mathrm{NS}} w_{\mathrm{N}} w_{\mathrm{S}}+P_{\mathrm{OS}} w_{\mathrm{O}} w_{\mathrm{S}}+ \\
& P_{\mathrm{Ti}} w_{\mathrm{Ti}}+P_{\mathrm{Nb}} w_{\mathrm{Nb}}+P_{\mathrm{B}} w_{\mathrm{B}}
\end{aligned}
$$

In the above equation, the second-order term and cross term for titanium, niobium and boron were ignored since these elements are not strong surface active elements. For the same reason the interaction on the surface tension between $\mathrm{Ti}-\mathrm{O}$ or $\mathrm{Ti}-\mathrm{N}$ also can be ignored although the titanium has strong interaction with oxygen and nitrogen in bulk phase in chemical thermodynamics point of view.

The parameters, $P_{\mathrm{i}}, P_{\mathrm{j}}, P_{\mathrm{ij}}$, could be obtained from the surface tension of Fe-i-j ternary system. For example, parameters, $P_{\mathrm{N}}, P_{\mathrm{O}}, P_{\mathrm{NN}}, P_{\mathrm{OO}}$ and $P_{\mathrm{NO}}$ could be obtained by determine the parameters that make Eq. (3) give the best fit to the experimental surface tension for $\mathrm{Fe}-\mathrm{N}-\mathrm{O}$ system $^{5)}$ as a function of concentration. However, there are some difficulties in assigning values to all the parameters from only the surface tension of ternary system due to the lack of experimental data. In the present paper, it has been attempted to derive the parameters from multicomponent system as well in addition to ternary (or binary) system. By using the surface tension data for the multicomponent system, the all parameters for the elements which is included in the system can be determined at same time by fitting. In the following calculations, a commercial software (Mathematica ver. 7.0, Wolfram Research) was used to determine the parameter values that make the equation for the surface tension (Eq. (6)) give the best fit to the surface tension data as a function of concentration.

Firstly, the parameters $P_{\mathrm{O}}$ and $P_{\mathrm{OO}}$ were determined from the surface tension of $\mathrm{Fe}-\mathrm{O}$ binary system at $1823 \mathrm{~K}^{5,6)}$ by

Table 5. Interaction parameters for multicomponent dilute solution (low carbon steel) at $1848 \mathrm{~K}$.

\begin{tabular}{cc}
\hline Parameter & Values at $1848 \mathrm{~K}$ \\
\hline$\sigma_{\mathrm{Fe}}$ & 1.945 \\
$P_{\mathrm{N}}$ & -7.029 \\
$P_{\mathrm{O}}$ & -19.45 \\
$P_{\mathrm{S}}$ & -2.289 \\
$P_{\mathrm{NN}}$ & -3.145 \\
$P_{\mathrm{OO}}$ & 102.1 \\
$P_{\mathrm{SS}}$ & 1.421 \\
$P_{\mathrm{NO}}$ & 126.6 \\
$P_{\mathrm{NS}}$ & 0 \\
$P_{\mathrm{OS}}$ & 15.74 \\
$P_{\mathrm{Ti}}$ & $-7.137 \times 10^{-1}$ \\
$P_{\mathrm{Nb}}$ & $7.857 \times 10^{-2}$ \\
$P_{\mathrm{B}}$ & $-7.106 \times 10^{-2}$ \\
\hline
\end{tabular}

fitting the experimental values to the following equation which corresponds to Eq. (3).

$$
\sigma=\sigma_{\mathrm{Fe}}+P_{\mathrm{O}} w_{\mathrm{O}}+P_{\mathrm{OO}} w_{\mathrm{O}}^{2}
$$

The surface tension of pure iron, $\sigma_{\mathrm{Fe}}$, at $1823 \mathrm{~K}$ was determined in advance from the literature ${ }^{5,7)}$ by linear fitting of surface tension against temperature.

Secondly, the parameters $P_{\mathrm{S}}, P_{\mathrm{SS}}$ and $P_{\mathrm{OS}}$ as well as $P_{\mathrm{N}}$, $P_{\mathrm{NN}}$ and $P_{\mathrm{NO}}$ were determined from the surface tension for $\mathrm{Fe}-\mathrm{O}-\mathrm{S}$ ternary system at $1873 \mathrm{~K}^{8,9)}$ and $\mathrm{Fe}-\mathrm{N}-\mathrm{O}$ ternary system at $1823 \mathrm{~K},{ }^{5)}$ respectively, by fitting the experimental values with the Eq. (3). The surface tension of pure iron at $1873 \mathrm{~K}$ and $1823 \mathrm{~K}$ were calculated from the literature data $^{5,7)}$ and used for the fitting. After that, the other parameters, $P_{\mathrm{Ti}}, P_{\mathrm{Nb}}$ and $P_{\mathrm{B}}$, were determined from the surface tension at $1848 \mathrm{~K}$ for synthesized multicomponent alloy ${ }^{3)}$ by fitting the experimental values to the Eq. (3). All the above parameters were regarded valid at $1848 \mathrm{~K}$. The temperature difference was neglected due to the lack of experimental data. The measurement error of the surface tension by sessile drop method is about $\pm 3 \%{ }^{10)}$ which is corresponding to about $\pm 60 \times 10^{-3} \mathrm{~N} \cdot \mathrm{m}^{-1}$ in the present system. On the other hand, according to the literature data for the surface tension of pure iron which is used in the present paper, ${ }^{5,7)}$ the temperature coefficient for the surface tension of pure iron is $-0.99 \times 10^{-3} \mathrm{~N} \cdot \mathrm{m}^{-1} \cdot \mathrm{K}^{-1}$. If it is assumed that this value is valid for the present systems as well, the temperature difference in the present calculation $( \pm 25 \mathrm{~K})$ is corresponding to $\pm 25 \times 10^{-3} \mathrm{~N} \cdot \mathrm{m}^{-1}$ in surface tension difference. Hence, considering the experimental error, the temperature difference could be negligible in the present system.

Regarding the parameter $P_{\mathrm{NS}}$, it was found that the value was not reliable due to the fact that the $P_{\mathrm{NS}}$ value became positive when the surface tension of $\mathrm{Fe}-\mathrm{O}-\mathrm{S}$ system by Ogino et $a l .{ }^{8)}$ was used for the calculation. On the other hand, it became negative value when the data by Gupt et al. ${ }^{9)}$ was used. At this stage, it is not adequate to evaluate the $P_{\mathrm{NS}}$ value accurately. Eventually, data sets of both Ogino et al. ${ }^{8)}$ and Gupt et $a l .{ }^{9)}$ are used for the calculation and $P_{\mathrm{NS}}$ value was put as zero (influence of the interaction between nitrogen and sulfur on the surface tension was ignored). Then the parameters, $P_{\mathrm{Ti}}, P_{\mathrm{Nb}}$ and $P_{\mathrm{B}}$ were determined that make the equation for the surface tension give the best fit to the surface tension data for the multicomponent system which contain titanium, niobium and boron ${ }^{3)}$ as a function of concentration. Since the parameters $P_{\mathrm{Ti}}, P_{\mathrm{Nb}}$ and $P_{\mathrm{B}}$ were fixed in the final step, the influence of neglect for the interaction between nitrogen and sulfur $\left(P_{\mathrm{NS}}=0\right)$, temperature difference and measurement errors were dumped into these parameters.

The values of the parameter at $1848 \mathrm{~K}$ are summarized in Table 5. The unit of surface tension is $\mathrm{N} \cdot \mathrm{m}^{-1}$.

\subsection{Parameters for the Surface Tension of 16 mass $\% \mathrm{Cr}$ SUS}

The surface tension of $16 \mathrm{Cr}$ SUS was also estimated using the surface thermodynamic model. ${ }^{1)}$ Due to the lack of experimental data to determine the parameters for surface tension model, only four elements, iron, chromium, oxygen and sulfur, were taken into account for the calculation. Regarding the $16 \mathrm{Cr}$ SUS, the calculation by the model aimed to estimate the surface tension with only strong sur- 
Table 6. Interaction parameters for $\mathrm{Fe}-16$ mass $\% \mathrm{Cr}-\mathrm{O}-\mathrm{S}$ system at $1823 \mathrm{~K}$.

\begin{tabular}{cc}
\hline Parameter & Values at $1823 \mathrm{~K}$ \\
\hline$\sigma_{\mathrm{Fe} 16 \mathrm{Cr}}$ & 1.750 \\
$P_{\mathrm{O}}^{\prime}$ & -19.44 \\
$P_{\mathrm{S}}^{\prime}$ & -6.675 \\
$P_{\mathrm{OO}}^{\prime}$ & 172.0 \\
$P_{\mathrm{SS}}^{\prime}$ & 20.97 \\
$P_{\mathrm{OS}}^{\prime}$ & 12.50 \\
\hline
\end{tabular}

face active elements. The influence of the other elements on the surface tension will not be assessed in the present calculation due to the simplification. The influence of the other elements on the surface tension are dumped into the parameters for oxygen and sulfur in the present calculation.

In this system, the $\mathrm{Cr}$ cannot be regarded as a dilute solute. Therefore the $\mathrm{Fe}-16$ mass\% $\mathrm{Cr}$ was treated as a "pure element". Then the surface tension of 16 mass $\% \mathrm{Cr}$ SUS can be described by Eq. (8) which corresponds to Eq. (3).

$$
\sigma=\sigma_{\mathrm{Fe} 16 \mathrm{Cr}}+P_{\mathrm{O}}^{\prime} w_{\mathrm{O}}+P_{\mathrm{S}}^{\prime} w_{\mathrm{S}}+P_{\mathrm{OO}}^{\prime} w_{\mathrm{O}}^{2}+P_{\mathrm{SS}}^{\prime} w_{\mathrm{S}}^{2}+P_{\mathrm{OS}}^{\prime} w_{\mathrm{O}} w_{\mathrm{S}}
$$

where $\sigma_{\mathrm{Fe} 16 \mathrm{Cr}}$ : the surface tension of $\mathrm{Fe}-16$ mass $\% \mathrm{Cr}, P_{\mathrm{i}}^{\prime}$ : Parameter (Coefficient) for the equation on surface tension of $\mathrm{Fe}-16$ mass $\% \mathrm{Cr}-\mathrm{O}-\mathrm{S}$ system by the surface thermodynamic model and $w_{\mathrm{i}}$ : the concentration of component $\mathrm{i}$ (mass \%).

New interaction parameters, $P^{\prime}$, in the Eq. (8) were determined by fitting the surface tension values for $\mathrm{Fe}-16$ mass $\% C r-O-S$ system at $1823 \mathrm{~K}^{11,12)}$ against concentration using Eq. (8). The obtained interaction parameters are shown in Table 6. The unit of surface tension is $\mathrm{N} \cdot \mathrm{m}^{-1}$.

\section{Discussion}

\subsection{Calculated Surface Tension}

The experimental surface tension values could be described by single equation. It implies also that the contribution of weak surfactants such as titanium, boron and niobium to the surface tension can be described by considering only first order terms.

The calculated surface tension values by Eq. (6) are plotted against experimental data which was used for the determination of parameters in Eq. (6) (Fig. 2). The data except for Ref. 13) was used for the determination of parameters. As shown in the Table 5, the parameters for Eq. (6) are valid at $1848 \mathrm{~K}$ and the surface tension of pure iron, $\sigma_{\mathrm{Fe}}$, at 1843 $\mathrm{K}$ is used. Therefore the calculation results on $\mathrm{Fe}-\mathrm{O}-\mathrm{S}$ system which measured at higher temperature $(1873 \mathrm{~K})$ are overestimated as can be seen from Fig. 2. Further, The data by Ogino et $a l .{ }^{8)}$ are deviated from the calc. =exp. line since the high number of data points by Gupt et al. ${ }^{9)}$ were used together with the data by Ogino et al. to determine the parameters by fitting.

The disagreement between the experimental and calculated values may become less by increasing the order of the equation for the surface tension since it is still possible to describe the parameters, $P$, by thermodynamic parameters

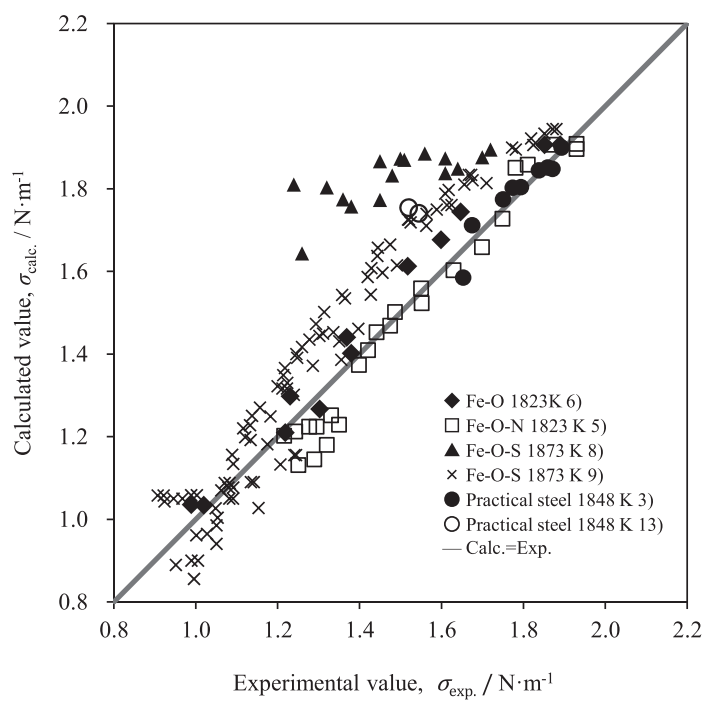

Fig. 2. Experimental surface tension value vs. Calculated surface tension value.

even if the order of the equation is increased. However, an appropriate order should be second order for the reliability of the experimental data.

It is to be noted that the degree of agreement between the experimental and calculated values can be improved if accurate experimental data for ternary system could be generated in the future. It is especially important to obtain the reliable measurement values of $\mathrm{Fe}-\mathrm{O}-\mathrm{S}$ and $\mathrm{Fe}-\mathrm{N}-\mathrm{S}$ systems (and $\mathrm{Fe}-\mathrm{O}-\mathrm{N}$ system, if possible) for the surface tension model since the parameters for these strong surface active elements is sensitive against surface tension. As mentioned, the measurement error of surface tension is about $\pm 60 \times 10^{-3} \mathrm{~N} \cdot \mathrm{m}^{-1}$. Hence, it is desired to determine the parameter values to make the average error of calculated surface tension value become less than $\pm 60 \times 10^{-3} \mathrm{~N} \cdot \mathrm{m}^{-1}$.

In order to verify the validity of the surface tension model, surface tension of two different practical steels, ${ }^{13)}$ which are not used for the determination of parameters, were calculated from the composition by using the surface tension model. The data is plotted in the Fig. 2 as open circles. The calculated values show reasonable agreement with the experimental data. As described before, the $P_{\mathrm{NS}}=0$ is applied and the influence of neglect for the interaction between nitrogen and sulfur is corrected by the parameters for titanium, niobium, and boron. In the present case, these practical steels contain high sulfur (0.02 mass $\%)$ and nitrogen $(0.01$ mass $\%$ ), and the amount of titanium, niobium and boron are not analyzed except for the titanium amount for one of them which has lower calculated and higher experimental value. Therefore the correction of the influence of neglect for the interaction between nitrogen and sulfur by $P_{\mathrm{Ti}}, P_{\mathrm{Nb}}$ and $P_{\mathrm{B}}$ terms is not sufficient. As a results, the calculated values became higher than the experimental values. However the surface tension will not be increased in total unless the amount of niobium become same level with boron or ten times of titanium as can be seen from parameter values, $P_{\mathrm{Ti}}$, $P_{\mathrm{Nb}}, P_{\mathrm{B}}$ in Table 5 .

The estimation of the surface tension by the model is still rough. However, there are some findings and advantages of the model. Generally speaking, it is difficult to know the 
influence of a specific species on the surface tension from the experimental results in the case of multicomponent systems when the concentrations of two or more species are changed simultaneously. However, it is possible to distinguish the contribution of each element to the surface tension by using the model. For example, in the experiment, the surface tension of a commercial steel seems to be decreased with increasing of niobium. ${ }^{3)}$ However the concentration of a surface active element, oxygen, was also increased. Therefore, it was impossible to evaluate the net effect of niobium. However, it is possible to evaluate the net effect of niobium on the surface tension by applying the surface tension model. As can be seen from the parameters in Table 5 and Eq. (6), the parameter for niobium, $P_{\mathrm{Nb}}$, has a positive value. Therefore, it can be concluded that the surface tension is increased with increasing of niobium.

\subsection{Validation of the Parameters}

In the previous paper, ${ }^{1)}$ it has been revealed that the surface tension can be described as a function of concentration and the coefficients (parameters) have thermodynamical meaning. At the present stage, it may difficult to calculate the parameters $P_{\mathrm{i}}, P_{\mathrm{ii}}$ and $P_{\mathrm{ij}}$ individually with thermodynamics or interfacial chemistry. Therefore the parameters must be determined by the measurements of surface tension. However, it is still possible to discuss the thermodynamical meaning of obtained parameter values as follows qualitatively.

As can be seen from Eqs. (2) and (3),

$$
P_{\mathrm{i}}=k \cdot \frac{M_{\mathrm{Fe}}\left(\mu_{\underline{\mathrm{i}}}^{\ominus^{\prime}}-\mu_{\underline{\mathrm{i}}}^{\ominus}\right)}{100 M_{\mathrm{i}} R T}
$$

The chemical potential of bulk phase, $\mu_{i}$ and the chemical potential of the phase including surface, $\mu_{\underline{1}}^{\prime}$, in the above equation can be expressed as follows,

$$
\begin{aligned}
& \mu_{\underline{i}}=\mu_{\underline{i}}^{\ominus}+R T \ln \left(a_{\mathrm{i}}\right) . \\
& \mu_{\underline{\mathrm{i}}}^{\prime}=\mu_{\underline{\mathrm{i}}}^{\ominus^{\prime}}+R T \ln \left(a_{\mathrm{i}}^{\prime}\right)
\end{aligned}
$$

where $a_{\mathrm{i}}$ is activity of component $\mathrm{i}$ and prime denotes the corresponding properties of the phase including surface phase.

At equilibrium state, $\mu_{\mathrm{i}}=\mu_{\mathrm{i}}^{\prime}$.

Therefore,

$$
\mu_{\underline{\mathrm{i}}}^{\ominus^{\prime}}-\mu_{\underline{\mathrm{i}}}^{\ominus}=R T \ln \left(a_{\mathrm{i}}\right)-R T \ln \left(a_{\mathrm{i}}^{\prime}\right)
$$

In the case of positive adsorption, $a_{\mathrm{i}}<a_{\mathrm{i}}^{\prime}$.

Hence sign of $\mu_{\mathrm{i}}^{\ominus^{\prime}}-\mu_{\mathrm{i}}^{\ominus}$, in other words, sign of $P_{\mathrm{i}}$ must be negative. Consequently, it is reasonable that the parameters $P_{\mathrm{i}}$ which obtained by curve fitting based on the experimental values of surface tension (Tables 5 and 6) have negative values.

Regarding the parameters $P_{\mathrm{ii}}$ and $P_{\mathrm{ij}}$, it can be described as follows as can be seen from Eqs. (2) and (3).

$$
P_{\mathrm{ii}}=k \cdot \frac{2.303 M_{\mathrm{Fe}}}{100 M_{\mathrm{i}}}\left(e_{\mathrm{i}}^{\mathrm{i}^{\prime}}-e_{\mathrm{i}}^{\mathrm{i}}\right)
$$

and

$$
P_{\mathrm{ij}}=k \cdot \frac{2.303 M_{\mathrm{Fe}}}{100} \cdot\left\{\frac{\left(e_{\mathrm{i}}^{\mathrm{j}^{\prime}}-e_{\mathrm{i}}^{\mathrm{j}}\right)}{M_{\mathrm{i}}}+\frac{\left(e_{\mathrm{j}}^{\mathrm{i}^{\prime}}-e_{\mathrm{j}}^{\mathrm{i}}\right)}{M_{\mathrm{j}}}\right\} \ldots \ldots
$$

$$
\begin{aligned}
& \quad \frac{e_{\mathrm{i}}^{\mathrm{j}^{\prime}}-e_{\mathrm{i}}^{\mathrm{j}}}{M_{\mathrm{i}}}+\frac{e_{\mathrm{j}}^{\mathrm{i}{ }^{\prime}}-e_{\mathrm{j}}^{\mathrm{i}}}{M_{\mathrm{j}}} \text { in the Eq. (14) may be arranged as } \\
& \frac{2}{M_{\mathrm{i}}}\left(e_{\mathrm{i}}^{\mathrm{j}^{\prime}}-e_{\mathrm{i}}^{\mathrm{j}}\right) \text { since } e_{\mathrm{j}}^{\mathrm{i}}=\frac{230 M_{\mathrm{j}} e_{\mathrm{i}}^{\mathrm{j}}+M_{\mathrm{i}}-M_{\mathrm{j}}}{230 M_{\mathrm{i}}} \\
& \text { and } e_{\mathrm{j}}^{\mathrm{i}^{\prime}}=\frac{230 M_{\mathrm{j}} e_{\mathrm{i}}^{\mathrm{j}^{\prime}}+M_{\mathrm{i}}-M_{\mathrm{j}}}{230 M_{\mathrm{i}}} \text {. }
\end{aligned}
$$

Hence, Eq. (14) may be arranged as follows:

$$
P_{\mathrm{ij}}=k \cdot \frac{2.303 M_{\mathrm{Fe}}}{100} \cdot\left\{\frac{2 \cdot\left(e_{\mathrm{i}}^{\mathrm{j}^{\prime}}-e_{\mathrm{i}}^{\mathrm{j}}\right)}{M_{\mathrm{i}}}\right\}
$$

Now,

$$
\begin{aligned}
\log f_{\mathrm{i}}^{\prime}-\log f_{\mathrm{i}} & =e_{\mathrm{i}}^{\mathrm{i}^{\prime}} w_{i}+\sum_{i \neq j} e_{\mathrm{i}}^{\mathrm{j}^{\prime}} w_{j}-e_{\mathrm{i}}^{\mathrm{i}} w_{i}-\sum_{i \neq j} e_{\mathrm{i}}^{\mathrm{j}} w_{j} \\
& =\left(e_{\mathrm{i}}^{\mathrm{i}^{\prime}}-e_{\mathrm{i}}^{\mathrm{i}}\right) w_{i}+\sum_{i \neq j}\left(e_{\mathrm{i}}^{\mathrm{j}^{\prime}}-e_{\mathrm{i}}^{\mathrm{j}}\right) w_{j}
\end{aligned}
$$

where $f_{\mathrm{i}}$ is the activity coefficient of component $\mathrm{i}$.

In the case of positive adsorption, $\log f_{\mathrm{i}}^{\prime}-\log f_{\mathrm{i}}>0$. Hence it could be concluded that the positive $P_{\mathrm{ii}}\left(e_{\mathrm{i}}^{\mathrm{i}{ }^{\prime}}-e_{\mathrm{i}}^{\mathrm{i}}>0\right)$ and $P_{\mathrm{ij}}\left(e_{\mathrm{i}}^{\mathrm{j}^{\prime}}-e_{\mathrm{i}}^{\mathrm{j}}>0\right)$ values which obtained by curve fitting based on the experimental values of surface tension are reasonable. The $P_{\mathrm{NN}}$ which has slightly positive value is only exception. This ought to be experimental error of the surface tension measurements.

\subsection{Mukai-value and the Number of Entrapped Bub- bles}

The driving force for the fine bubble entrapment by the solidifying shell can be assessed by the Mukai-value, $M$ as mentioned in the beginning of this paper. In the previous paper, ${ }^{2)}$ the Mukai-value was calculated using Eq. (4) with the $\mathrm{d} \sigma / \mathrm{d} w_{\mathrm{i}}$ value at $w_{\mathrm{i}}=0$. It is assumed that the concentration of element $i$ is decreased linearly in the boundary layer and the surface tension is increased linearly toward the bulk. As a results, the $\mathrm{d} \sigma / \mathrm{d} w_{\mathrm{i}}$ value on the bulk side was overestimated and the Mukai-value was also overestimated. Especially, the Mukai-value for Low Al stainless steel was higher in the previous calculation and the value became more than $2 \mathrm{~N} \cdot \mathrm{m}^{-1}$. Mukai-value, which corresponds to the surface tension difference, cannot have such high value since the surface tension value itself is less than $2 \mathrm{~N} \cdot \mathrm{m}^{-1}$ in the present system.

The Mukai values derived in the previous paper was useful as a relative scale to evaluate the difference of the driving force for the movement of bubbles by composition difference. In this paper, an attempt was made to evaluate the Mukai-value quantitatively. In this paper, the Mukai values were calculated using Eq. (5) with the estimated surface tension values by the surface tension model. The surface tension at bulk $\left(\sigma\left(w_{\mathrm{i}}\right)\right)$ in the Eq. (5) was estimated from the composition shown in Tables 1 and 2 by using Eq. (6) for low carbon steels and Eq. (8) for 16 Cr SUS stainless steels. To estimate the surface tension at the solidifying interface in the Eq. (5) from the steel composition, the composition at the solidifying interface was estimated by using following equilibrium partition coefficient, $k_{0}{ }^{\mathrm{i}}: k_{0}^{\mathrm{S}}=0.05, k_{0}^{\mathrm{Ti}}=0.40$, $k_{0}{ }^{\mathrm{N}}=0.28$ and $k_{0}^{\mathrm{O}}=0.1^{14-17)}$ for low carbon steel. The same 
Table 7. The composition at bulk and solidifying interface (low carbon steel).

\begin{tabular}{lcccccc}
\hline Element & Low carbon (1) Bulk & $\begin{array}{c}\text { Low carbon(1) } \\
\text { Solidifying interface }\end{array}$ & Low carbon (2) Bulk & $\begin{array}{c}\text { Low carbon (2) } \\
\text { Solidifying interface }\end{array}$ & Low carbon (3) Bulk & $\begin{array}{c}\text { Low carbon (3) } \\
\text { Solidifying interface }\end{array}$ \\
\hline $\mathrm{S}$ & 0.0088 & 0.1760 & 0.0138 & 0.2760 & 0.0142 & 0.2840 \\
$\mathrm{Ti}$ & 0.0000 & 0.0000 & 0.0009 & 0.0023 & 0.0586 & 0.1465 \\
$\mathrm{~N}$ & 0.0023 & 0.0082 & 0.0035 & 0.0125 & 0.0029 & 0.0104 \\
Free O & 0.0001 & 0.0010 & 0.0001 & 0.0010 & 0.0001 & 0.0010 \\
\hline
\end{tabular}

Table 8. The composition at bulk and solidifying interface (low Al stainless steel).

\begin{tabular}{|c|c|c|c|c|c|c|}
\hline Element & Low Al (1) Bulk & $\begin{array}{l}\text { Low Al (1) } \\
\text { Solidifying interface }\end{array}$ & Low Al (2) Bulk & $\begin{array}{c}\text { Low Al (2) } \\
\text { Solidifying interface }\end{array}$ & Low Al (3) Bulk & $\begin{array}{c}\text { Low Al (3) } \\
\text { Solidifying interface }\end{array}$ \\
\hline S & 0.0085 & 0.1700 & 0.0087 & 0.1740 & 0.0071 & 0.1420 \\
\hline $\mathrm{N}$ & 0.0363 & 0.1296 & 0.0331 & 0.1182 & 0.0324 & 0.1157 \\
\hline Free $\mathrm{O}$ & 0.0020 & 0.0200 & 0.0045 & 0.0450 & 0.0035 & 0.0350 \\
\hline
\end{tabular}

Table 9. The composition at bulk and solidifying interface (medium and high Al stainless steel).

\begin{tabular}{|c|c|c|c|c|c|c|}
\hline Element & Medium Al (1) Bulk & $\begin{array}{c}\text { Medium Al (1) } \\
\text { Solidifying interface }\end{array}$ & Medium Al (2) Bulk & $\begin{array}{c}\text { Medium Al (2) } \\
\text { Solidifying interface }\end{array}$ & High Al Bulk & $\begin{array}{c}\text { High Al } \\
\text { Solidifying interface }\end{array}$ \\
\hline S & 0.0014 & 0.0280 & 0.0021 & 0.0420 & 0.0013 & 0.0260 \\
\hline $\mathrm{N}$ & 0.0284 & 0.1014 & 0.0030 & 0.0107 & 0.0111 & 0.0396 \\
\hline Free $\mathrm{O}$ & 0.0005 & 0.0050 & 0.0004 & 0.0040 & 0.0003 & 0.0030 \\
\hline
\end{tabular}

Table 10. Calculated surface tension and Mukai-values.

\begin{tabular}{cccc}
\hline Steel grade & $\begin{array}{c}\text { Surface tension } \\
\text { at bulk }\left[\mathrm{N} \cdot \mathrm{m}^{-1}\right]\end{array}$ & $\begin{array}{c}\text { Surface tension } \\
\text { at solidifying } \\
\text { interface }\left[\mathrm{N} \cdot \mathrm{m}^{-1}\right]\end{array}$ & $\begin{array}{c}\text { Mukai-value }\left[\mathrm{N} \cdot \mathrm{m}^{-1}\right] \\
\left(\sigma\left(w_{\mathrm{i}}\right)-\sigma\left(w_{\mathrm{i}} / k_{\mathrm{o}}{ }^{\mathrm{i}}\right)\right)\end{array}$ \\
\hline LC (1) & 1.907 & 1.513 & 0.394 \\
LC (2) & 1.887 & 1.318 & 0.569 \\
LC (3) & 1.849 & 1.218 & 0.631 \\
\hline
\end{tabular}

equilibrium partition coefficients were used to estimate the concentration of each element at solidifying interface of stainless steels as well due to the lack of the data on equilibrium partition coefficients for $16 \mathrm{Cr}$ SUS. Regarding the equilibrium partition coefficients for oxygen, $k_{0} \mathrm{O}=0.02 \sim 0.1$ have been reported. ${ }^{16-22)}$ In the present system, aluminium oxide will be thermodynamically formed at solidifying interface when $k_{0}^{\mathrm{O}}=0.02$ is applied since the oxygen concentration at solidifying interface becomes high. However the aluminium oxide was not formed in the practical operation. Hence $k_{0}^{\mathrm{O}}=0.1$, which does not form aluminium oxide thermodynamically, seems to be reasonable value in the present case. The maximum oxygen concentration at solidifying interface becomes 0.045 mass $\%$ with $k_{0}^{\mathrm{O}}=0.1$ and aluminium oxide will not be formed since the oxygen can be supersaturated. According to Hilty and Crafts, ${ }^{23)}$ and Novokhatskiy and Belov, ${ }^{24)}$ the oxygen could be supersaturated at least 0.045 mass $\%$.

The composition at bulk and solidifying interface which used to estimate the surface tension is summarized in Tables 7-9. The surface tension at $1823 \mathrm{~K}$ and the Mukai values calculated from the surface tension difference between bulk and solidifying interface (Eq. (5)) are shown in Tables $\mathbf{1 0}$ and $\mathbf{1 1}$

The Mukai-value calculated using Eq. (5) with the surface
Table 11. Calculated surface tension and Mukai value for stainless steel.

\begin{tabular}{lccc}
\hline Steel grade & $\begin{array}{c}\text { Surface tension } \\
\text { at bulk }\left[\mathrm{N} \cdot \mathrm{m}^{-1}\right]\end{array}$ & $\begin{array}{c}\text { Surface tension } \\
\text { at solidifying } \\
\text { interface }\left[\mathrm{N} \cdot \mathrm{m}^{-1}\right]\end{array}$ & $\begin{array}{c}\text { Mukai-value }\left[\mathrm{N} \cdot \mathrm{m}^{-1}\right] \\
\left(\sigma\left(w_{\mathrm{i}}\right)-\sigma\left(w_{\mathrm{i}} / k_{\mathrm{o}}{ }^{\mathrm{i}}\right)\right)\end{array}$ \\
\hline Low Al (1) & 1.657 & 0.944 & 0.713 \\
Low Al (2) & 1.610 & 0.795 & 0.815 \\
Low Al (3) & 1.638 & 0.817 & 0.821 \\
Medium Al (1) & 1.731 & 1.488 & 0.243 \\
Medium Al (2) & 1.728 & 1.434 & 0.294 \\
High Al & 1.736 & 1.535 & 0.201 \\
\hline
\end{tabular}

tension model became smaller compared with previous values $^{2)}$ calculated using Eq. (4) with $k_{0}^{\mathrm{O}}=0.1$ and physically reasonable Mukai-values could be obtained. In the present calculation. the surface tension difference $\left(\sigma\left(w_{\mathrm{i}}\right)-\sigma\left(w_{\mathrm{i}} / k_{\mathrm{o}}{ }^{\mathrm{i}}\right)\right)$ becomes between 0.201 and $0.821 \mathrm{~N} \cdot \mathrm{m}^{-1}$. In the previous work, the Mukai-value was not calculated from the surface tension difference directly but calculated by using surface tension gradient (Eq. (4)). Therefore the Mukai-value became larger than reasonable surface tension difference for some cases. By calculating the Mukai-value from the surface tension difference directly (Eq. (5)) by using the surface tension model, Mukai-value could be determined quantitatively.

In order to verify the validity of the Mukai-value, the number of entrapped bubbles was plotted against Mukaivalue. Figures 3 and $\mathbf{4}$ show the results for low carbon steel and stainless steel, respectively. As can be seen from these figures, the number of bubbles is increased with increasing of Mukai-value and it shows linear relationship. The force act on the bubbles (ex. buoyancy, force by convection) can be regarded as same except for the force caused by the surface tension gradient since the casting condition is same. 


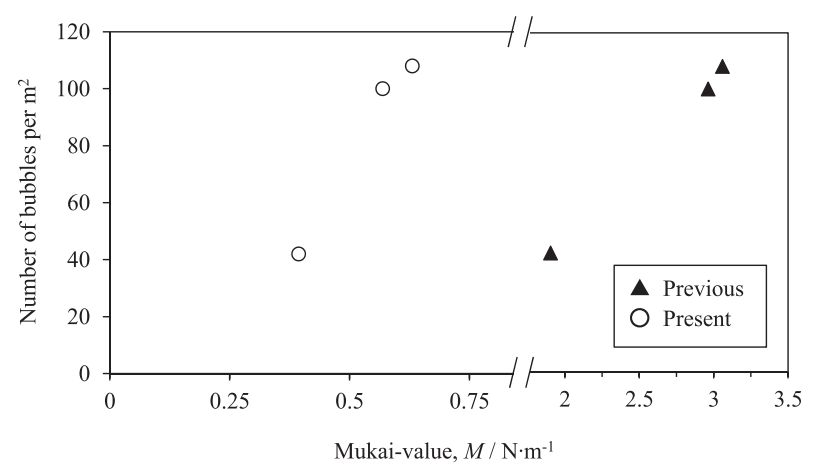

Fig. 3. Relationship between Mukai-value, $M$ and number of bubbles in the low-carbon slabs.

Hence it is possible to correlate the movement of bubbles directly to the Mukai-value. The results indicate that the Mukai-value (driving force for the fine bubble entrapment by the solidifying shell) are proportional to the number of captured bubbles, which shows the Mukai value is a valid parameter to evaluate the bubble entrapment.

The number of bubbles, which radius is larger than 0.1 $\mathrm{mm}$, becomes zero below a certain critical Mukai-value, $M_{\mathrm{cr}}=0.24$ under the present casting conditions in the low carbon steel case. The smaller bubbles can be regarded as harmless bubbles from the casting quality point of view and practically the influence of such small bubbles can be negligible. In the case of $16 \mathrm{Cr}$ SUS, the critical Mukai-value becames 0.1 under the present casting conditions. The difference of the critical value is due to the difference of concentration boundary layer thickness in front of the solidifying interface caused by the casting condition difference such as cooling speed and the difference of the counted bubble size. The crical Mukai-value will be varied by the following casting conditions as well: i) amount of argon, ii) casting speed, iii) condition of electromagnetic stirring, iv) shape of nozzle, v) width of slab, etc. since such operating conditions are not taken into account in the Mukai-value. The number of entrapped bubbles at a certain Mukai-value will be varied by changing above mentioned conditions.

It is, however, possible to propose guiding principles to suppress the number of defects caused by entrapped bubbles based on the Mukai-value which is derived for the same casting conditions to evaluate the influence of composition. As mentioned previously, the entrapment of the bubbles will be suppressed by introducing positive surface tension gradient toward the solidification interface or minimize the Mukaivalue. By combining it with the surface tension model for surface tension, a guideline to decrease the Mukai-value can be found theoreticaly with higher accuracy compared with previous calculation method. It could be possible to find out the elements which give smaller or negative Mukai-values by using the surface tension model. For example, as can be seen from Table 5, the niobium has positive surface tension gradient toward the solidification interface $\left(P_{\mathrm{Nb}}>0\right)$. Therefore, if the niobium is introduced in the system so that the concentration of niobium becomes higher toward the solidification interface, the Mukai-value will become smaller and the fine bubble capturing by the interface will be suppressed.

As shown in the relationship between the number of bub-

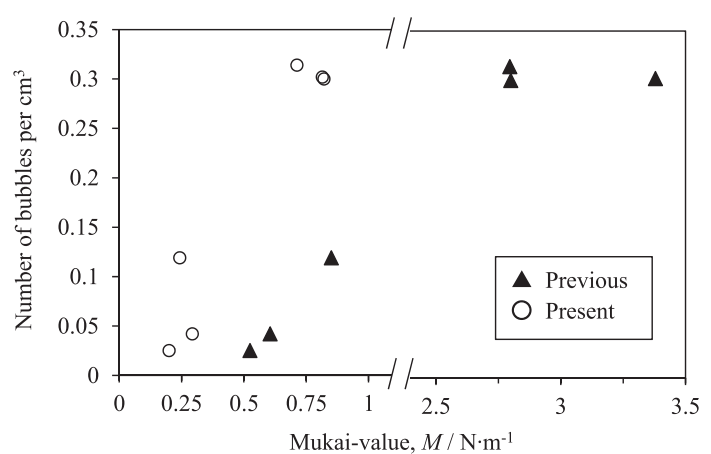

Fig. 4. Relationship between Mukai-value, $M$ and number of bubbles in the $16 \mathrm{Cr}$ SUS steels.

bles and the Mukai-value which obtained under the same casting condition, a linear relationship were found between these two factors. Hence, it is also possible to find out the influence of the casting condition on the bubble entrapment by changing the casting condition and investigate the relationship of these two factors in the same way. Such results will give useful information for the optimization of the process.

\section{Conclusion}

The surface tension of the multicomponent practical steels could be predicted by using the surface thermodynamic model with parameters derived from literature data. It was found that the influence of weak surfactants such as titanium, niobium and boron on the surface tension could be described by considering only first order terms. Further, the influence of a specific element on the surface tension was clarified based on the model.

The precise surface tension measurements for $\mathrm{Fe}-\mathrm{O}-\mathrm{S}$ and $\mathrm{Fe}-\mathrm{N}-\mathrm{S}$ systems (and $\mathrm{Fe}-\mathrm{O}-\mathrm{N}$ system, if possible) are essential to determine the parameters related to these elements more precisely for the model.

The Mukai-value, $M$ was calculated from the surface tension which is predicted by the surface tension model and applied for the evaluation of the entrapment behavior of the fine bubbles by solidifying shell. A guideline to suppress the fine bubble entrapment has been proposed based on the mechanism of the fine particle entrapment and the surface tension model. The bubble entrapment will be suppressed by introducing elements such as niobium which gives higher surface tension toward solidifying shell.

It can be concluded that the Mukai-value calculated by using the surface tension model is a useful parameter for the optimization of the process to suppress the number of defects caused by the bubble entrapment, mainly from the aspect of optimization of composition.

\section{Nomenclature}

A: Area

$A_{0}$ : Surface area of the system with one mole solution

$a_{\mathrm{i}}$ : Activity of component $\mathrm{i}$

$e_{\mathrm{i}}^{\mathrm{j}}$ : Interaction parameter in the bulk

$e_{\mathrm{i}}^{\mathrm{j}^{\prime}}$ : Interaction parameter in the phase which including surface

$F$ : Helmholz energy 
$f_{\mathrm{i}}$ : Activity coefficient of component $\mathrm{i}$

$k: \quad k=\left(R T / A_{0}\right) \cdot 10^{7}$

$k_{0}{ }^{\mathrm{i}}$ : Equilibrium partition coefficient of component $\mathrm{i}$

$M$ : Mukai value

$M_{\text {cr: }}$ Critical Mukai-value

$M_{\mathrm{i}}$ : Atomic weight of component i

$n_{\mathrm{i}}$ : Number of moles of component $\mathrm{i}$

$n_{\mathrm{i}}^{\mathrm{b}}$ : Number of moles of component $\mathrm{i}$ in the bulk phase

$n_{\mathrm{i}}^{\mathrm{s}}$ : Number of moles of component $\mathrm{i}$ in (or at) the surface (area).

$P^{l}$ : Pressure of liquid phase

$P_{\mathrm{i}}$ : Parameter (Coefficient) for the equation on surface tension by the surface thermodynamic model

$P_{\mathrm{i}}^{\prime}$ : Parameter (Coefficient) for the equation on surface tension of $\mathrm{Fe}-16$ mass $\% \mathrm{Cr}-\mathrm{O}-\mathrm{S}$ system by the surface thermodynamic model

$R$ : Gas constant

T: Temperature

$V$ : Volume

$V^{l}$ : Volume of liquid phase

$w_{\mathrm{i}}$ : Mass percent of the component $\mathrm{i}$

$\mu_{\mathrm{i}}$ : Chemical potential of component $\mathrm{i}$

$\mu_{\mathrm{i}}$ : Chemical potential of component $\mathrm{i}$ in the bulk phase

$\mu_{\mathrm{i}}^{\prime}$ : Chemical potential of component $\mathrm{i}$ in the phase including surface

$\mu_{i}^{\ominus}$ : Chemical potential of $i$ in the bulk for the standard state at $1 \mathrm{mass} \%$ i for a dilute, ideal solution

$\mu_{\mathrm{i}}^{\ominus^{\prime}}$ : Chemical potential of $\mathrm{i}$ in the phase which including surface for the standard state at 1 mass $\%$ i for a dilute, ideal solution $\sigma:$ Surface tension

$\sigma_{\mathrm{Fe}}:$ Surface tension of pure iron

$\sigma_{\mathrm{Fe} 16 \mathrm{Cr}}:$ Surface tension of $\mathrm{Fe} 16$ mass $\% \mathrm{Cr}$

\section{REFERENCES}

1) K. Mukai, T. Matsushita, K. C. Mills, S. Seetharaman and T. Furuzono: Metall. Mater. Trans. B, 39 (2008), 561.

2) K. Mukai and M. Zeze: Steel Res., 74 (2003), 131

3) K. Mukai, L. Zhong and M. Zeze: ISIJ Int., 46 (2006), 1810.

4) M. Zeze and K. Mukai: Proc. of 3rd Int. Cong. on the Science and Technology of Steelmaking (ICS2005), Warrendale, PA, USA, (2005), 873.

5) J. Zhu and K. Mukai: ISIJ Int., 38 (1998), 1039.

6) N. Takiuchi, T. Taniguchi, N. Shinozaki and K. Mukai: J. Jpn. Inst. Met., 55 (1991), 44

7) N. Takiuchi, T. Taniguchi, Y. Tanaka, N. Shinozaki and K. Mukai: J. Jpn. Inst. Met., 55 (1991), 180.

8) K. Ogino, K. Nogi and C. Hosoi: Tetsu-to-Hagané, 69 (1983), 1989.

9) K. M. Gupt, V. I. Vishkaryov and S. A. Bliznukov: Trans. Ind. Inst. Met., 29 (1976), 286.

10) K. Mukai: Kōon yūtai no kaimen butsuri kagaku, Agne, Tokyo, (2007), 86

11) K. Mukai, Z. Li and M. Zeze: Mater. Trans, 43 (2002), 1724.

12) Z. Li, M. Zeze and K. Mukai: Mater. Trans., 44 (2003), 2108.

13) R. F. Brooks and P. N. Quested: J. Mater. Sci., 40 (2005), 2233.

14) Handbook of Iron and Steel - I -, 3rd ed., ISIJ, Tokyo, (1981), 193.

15) Z. Morita and T. Tanaka: Tetsu-to-Hagané, 74 (1988), 1210.

16) A. Hays and J. Chipman: Trans. Am. Inst. Min. Metall. Eng., 135 (1939), 85.

17) W. A. Tiller: J. Iron Steel Inst., 192 (1959), 338.

18) J. Chipman: Basic Open Hearth Steelmaking, the American Institute of Mining and Metallurgical Engineering, New York, (1951), 644.

19) W. A. Fischer, H. Spitzer and M. Hishinuma: Arch. Eisenhüttenwes., 31 (1960), 365.

20) M. T. Hepworth, R. P. Smith and E. T. Turkdogan: Trans. Metall. Soc. AIME, 236 (1966), 1278

21) A. Kusano, K. Ito and K. Sano: Tetsu-to-Hagané, 54 (1968), 553.

22) F. Oeters and K. Rüttiger: Arch. Eisenhüttenswes., 40 (1969), 603.

23) D. C. Hilty and W. Crafts: J. Met., Trans. AIME, 188 (1950), 414.

24) I. A. Novokhatskiy and B. F. Belov: Izv. Akad. Nauk SSSR, 3 (1969), 15 . 